\title{
Treatment of Hepatocellular Carcinoma-associated Portal Vein Tumor Thrombus Using Combination of Portal Vein Stent Insertion and Endovascular Lodine-125 Seed-strip Implantation Followed by Transcatheter Arterial Chemoembolization- Sorafenib
}

Shuangxi Li

Lanzhou University First Affiliated Hospital

Baohua Li

Lanzhou University First Affiliated Hospital

Lei Li

Lanzhou University First Affiliated Hospital

Fangyu Xu

Lanzhou University First Affiliated Hospital

Xujun Yang

Lanzhou University First Affiliated Hospital

Wenhui Wang ( $470090101 @ q q . c o m$ )

Lanzhou University First Affiliated Hospital https://orcid.org/0000-0003-0861-2936

\section{Research}

Keywords: hepatocellular carcinoma, portal vein, transcatheter arterial chemoembolization, iodine-125, sorafenib

Posted Date: October 8th, 2020

DOI: https://doi.org/10.21203/rs.3.rs-88313/v1

License: (c) (1) This work is licensed under a Creative Commons Attribution 4.0 International License. Read Full License 


\section{Abstract}

\section{Background}

To evaluate the efficacy of portal vein stent (PVS) insertion and endovascular iodine-125 ( $\left.{ }^{125} \mid\right)$ seed-strip implantation followed by transcatheter arterial chemoembolization (TACE)-sorafenib (PVS- ${ }^{125}$ I-TACE-S) in patients with hepatocellular carcinoma (HCC)-associated portal vein tumor thrombus (PVTT).

Methods

This retrospective analysis was performed on 53 consecutive HCC patients with PVTT between May 2014 and July 2018. Twenty-eight patients were treated via PVS- ${ }^{125}$ I-TACE-S, and 25 patients were treated by TACE-S. Hepatic function, disease control, and overall survival (OS) in both groups were analyzed. The Albumin-Bilirubin (ALBI) score approach was used as a means of evaluating liver function, and a Cox regression analysis was utilized to determine which factors were linked to treatment outcomes.

Results

No preoperative differences in ALBI scores between groups $(P=0.724)$, nor did these scores at 1-month postoperative $(P=0.666)$. However, these scores were significantly different at $3(P=0.007)$ and 6 $(P=0.044)$ months postoperatively. PVS-125-TACE-S exhibiting higher rates of disease control $(71.4 \% \mathrm{vs.}$ $44.0 \%, P=0.043$ ) after 6 months of treatment and extended OS duration (342.0 vs 231.0 days, $P=0.007)$. A stratified analysis revealed OS in patients with type II PVTT did not differ significantly (313.0 vs. 321.0 days, $P=0.689$ ), but OS with type III did (344.0 vs. 226.0 days, $P=0.002)$. A multivariate analysis revealed that tumor size $>10 \mathrm{~cm}$ was independently predictive of poor prognosis $(P=0.002)$, whereas PVS- ${ }^{125}$ TACE-S was a predictor of a favorable patient prognosis $(\mathrm{P}=0.040)$.

Conclusion

PVS- ${ }^{125}$ I-TACE-S represents a potentially viable strategy for improving hepatic functionality, disease control, and OS in HCC with type III PVTT.

\section{Background}

Hepatocellular carcinoma (HCC), owing to the typical lack of early symptoms, is typically diagnosed when the disease has progressed to mid- or late-stage cancer. Approximately $44.0 \%-62.2 \%$ of patients exhibit macroscopic portal vein tumor thrombus (PVTT) when initially diagnosed [1,2]. The presence of PVTT can have a severe adverse effect on the hepatic blood supply, leading to decreased liver function and poor patient prognosis [3]. Median survival time of HCC patients without treatment affected by PVTT is reduced to $2.7-4.0$ months, as opposed to $10-24$ months in those without this comorbidity $[4,5]$.

At present, however, the optimal means of treating HCC complicated by PVTT remains uncertain. The Barcelona Clinic Liver Cancer (BCLC) group recommends that sorafenib be the therapeutic agent of 
choice for individuals with advanced HCC, irrespective of PVTT status. The Sorafenib Hepatocellular Carcinoma Assessment Randomized Protocol (SHARP trial), on which BCLC recommendations were based, however, suggests that vascular invasion is only present in $38.4 \%$ of HCC patients, and as such sorafenib alone may not necessarily confer improved survival to HCC patients with PVTT [6,7]. In addition, studies of Asian populations with vascular invasion or extrahepatic metastasis have suggested that sorafenib treatment is associated with poor overall survival (OS; median: 6.5 months) $[6,8]$. Indeed, consistent with this the Asia-Pacific guidelines recommend that HCC patients with PVTT undergo a multipronged antitumor treatment course that can include surgery, sorafenib, and radiotherapy or transcatheter arterial chemoembolization (TACE) as potential interventions [9]. There has been some research regarding the use of TACE-sorafenib (TACE-S) owing to observations that it exhibits therapeutic synergy when employed to treat HCC patients with PVTT provided there is good patency for the main portal vein or sufficient collateral circulation $[6,10,11]$. This combination is associated with some increase in patient survival, but overall the efficacy of this approach is limited, and a superior combination strategy is thus needed [12].

The use of a portal vein stent (PVS) to reduce portal vein occlusion has been explored in the context of PVTT, and its combination with iodine-125 $\left({ }^{125} \mathrm{I}\right)$ seed brachytherapy offers a potentially optimal means of improving PVTT management in HCC patients [13]. There are previous reports showing that this combination could decrease liver toxicity caused by TACE, and the synergic effect or abscopal effect offers better local control and OS [14,15]. Therefore, PVS insertion and endovascular ${ }^{125}$ I seed-strip $^{2}$ implantation followed by TACE-S has a strong scientific rationale [16]. In the present study, a retrospective analysis was conducted to assess the efficacy of this approach and the factors associated with patient outcomes.

\section{Methods}

This study received approval from the ethics committee of our institution, and all provided written informed consent before participating.

The institutional database used in this study was compiled using data from HCC patients with PVTT who underwent endovascular ${ }^{125}$ I seed-strip implantation followed by TACE-S (PVS-125I-TACE-S) or TACE-S from May 2014 to July 2018. For all patients, attending physicians had recommended TACE, sorafenib, TACE-S, or PVS-125I-TACE-S treatment, with final treatment approaches having been selected by the individual patients. Study inclusion criteria were as follows: 1) diagnosis with HCC and either type II or type III PVTT; 2) no previous local treatment for PVTT lesions; 3) An Eastern Cooperative Oncology Group (ECOG) performance of $0-2 ; 4$ ) Child-Pugh Class A or B. Study exclusion criteria were as follows: 1 ) complete portal vein occlusion with a lack of collateral circulation; 2) bleeding of the esophagus or gastric fundus; 3 ) intractable coagulation disorders; 4) macroscopic hepatic vein tumor thrombi or extrahepatic tumor metastasis; 5) a lack of baseline imaging results. 
A total of 64 consecutive HCC patients with PVTT were identified, including 7 patients that underwent simple TACE, and 1 patient treated with only sorafenib. In addition, preoperative enhanced CT/MRI scan results were not available for 2 patients, and 1 patient was lost to follow up. These patients were excluded from the study, leaving a final study population of 53 patients. A total of 28 of these patients were treated via PVS-125I-TACE-S (group A), while the remaining 25 patients were treated via TACE-S alone (group B).

In all patients, HCC diagnosis was confirmed either via histology or by a combination of two imaging approaches and elevated a-fetoprotein, as per the American Association guidelines [17]. Magnetic resonance imaging (MRI) or computed tomography $\mathrm{CT}$ ) was used to diagnose PVTT based on the presence of a low attenuation intraluminal mass that partially or fully occluded the portal vein or filled a portal vein defect [18]. Patients were stratified into Type I - IV PVTT according to Cheng's PVTT classification system based on the following criteria: Type I, tumor thrombi locating at or above portal vein segmental branches (second-order branches); Type II, tumor thrombi extending so as to involve the right or left portal vein (first-order branches); Type III, thrombi involving the main trunk of the portal vein; Type IV, invasion of the superior mesenteric vein or inferior vena cava [4].

\section{PVS combined with endovascular ${ }^{125}$ I seed-strip implantation}

The required number $(N)$ of ${ }^{125}$ I seeds was determined based upon the length $(L)$ of the obstructed portal vein segment in $\mathrm{mm}$ per the formula $\mathrm{N}=\mathrm{L} / 4.5+2$ (Shanghai GMS Pharmaceutical Co. Ltd) [13]. The ${ }^{125}$ I seeds used in this study were $0.8 \mathrm{~mm}$ in diameter, and $4.5 \pm 0.5 \mathrm{~mm}$ long, with $25.9 \mathrm{MBq}$ of radioactivity and a 59.4-day half-life, primarily emitting 27.4 and $31.4 \mathrm{keV} X$-rays and $35.5 \mathrm{keV} \gamma$-rays. Given the local tissue half-value thickness of $17 \mathrm{~mm}$, these seeds were associated with an initial dose rate of $7 \mathrm{cGy} / \mathrm{h}$. Prior to implantation, these seeds were loaded in a linear arrangement in a $4 \mathrm{Fr}$ flexible stiffening cannula (Boston Scientific Co., Ltd). With ultrasonic guidance, a Neff Percutaneous Access Set (Cook, Inc., IN, USA) was then used to puncture the patent second-order portal vein branch, after which both a vascular stent (diameter: 12-14 mm; length: 60-100 mm; Bard Peripheral Vascular Inc., AZ, USA) and ${ }^{125}{ }^{\text {I seed-strip }}$ were implanted in succession. After this, a 3-3 spring coil (Cook, Inc., Bloomington, Indiana) was used for blocking the intrahepatic puncture, and subcutaneous low-molecular-weight heparin (4100 IU; Hebei Changshan Biochemical Pharmaceutical Co, Ltd) was administered twice daily for a 5 day period, after which warfarin (Shanghai Sine Pharmaceutical Laboratories Co., Ltd) was administered orally in order to achieve the international normalized ratio of 2.0-2.5.

\section{TACE procedure}

In group A patients, TACE was conducted 3 - 7 days after PVS and seed implantation, whereas in group B patients this procedure was conducted directly. A 5-Fr hepatic-curve catheter (Terumo Corporation, Japanese) was placed into the celiac artery and then hepatic arterial angiography and indirect portography were performed. Next, a $2.7 \mathrm{Fr}$ microcatheter (Progreat ${ }^{\mathrm{TM}}$, Terumo, Tokyo, Japan) was placed into tumor-feeding arteries, and 5-20 mL lipiodol (Beijing Wh-Medical Apparatus and Instruments Co. 
Ltd) mixed with $50-75 \mathrm{mg} / \mathrm{m}^{2}$ doxorubicin hydrochloride (Shanghai Hisun Pfizer Pharmaceutical Co. $L t d)$ were injected into these arteries. If the entire $20 \mathrm{~mL}$ volume was administered without substantially impairing blood flow in these vessels, polyvinyl alcohol (PVA) particles (Hangzhou ALICON Pharmaceutical Science and Technology Co. Ltd) were used for vessel embolization, being administered until only a limited slow flow was evident. For those with arterioportal shunts, initial embolization using 350-1000 $\mu \mathrm{m}$ PVA particles was conducted prior to lipiodol/doxorubicin infusion in order to ensure shunt occlusion. TACE treatment was guided by imaging results.

\section{Sorafenib}

Three to seven days after TACE treatment, when liver function had stabilized, patients were administered 400 mg sorafenib (Nexavar®, Bayer HealthCare, Leverkusen, Germany) twice per day. In many patients suffering from grade 1 or 2 adverse events (AEs), or with serum bilirubin $>34.2 \mu \mathrm{mol} / \mathrm{L}$, sorafenib doses were reduced. In patients suffering grade 3 AEs or hyperbilirubinemia (serum bilirubin $>51.3 \mu \mathrm{mol} / \mathrm{L}$ ) events, sorafenib treatment was temporarily halted and was only resumed if $A E$ grades and serum bilirubin levels fell back within the acceptable treatment ranges. Patients were continuously administered sorafenib tablets until it was no longer possible to do so, even when disease progression was noted, or until death.

\section{Follow-up and treatment evaluation}

At 1 and 3 months post-operatively, patient follow-up was conducted, and it was then performed every three months thereafter. During follow-up, patients underwent physical examination, laboratory analyses, as well as contrast-enhanced abdominal MRI or CT. The Common Terminology Criteria for Adverse Events (CTCAE) v4.0 assessment was used to gauge any complications associated with treatment [8]. AlbuminBilirubin (ALBI) scores were used as an objective means of gauging liver function, with scores being calculated solely based upon albumin and bilirubin levels [ALBI score $=\log 10$ bilirubin $(\mu \mathrm{mol} / \mathrm{L}) \times 0.66)+$ (albumin ( $\mathrm{g} / \mathrm{L}) \times-0.0852)$ ] [17]. The modified Response Evaluation Criteria in Solid Tumors (mRECIST) criteria for $\mathrm{HCC}$ were used to gauge tumor responses, with possible responses including complete response (CR), partial response (PR), stable disease (SD), or progressive disease (PD) (Fig. 1). A disease control rate (DCR) after 6 months of treatment was then calculated based on the formula: $(\mathrm{CR}+\mathrm{PR}+\mathrm{SD}) /$ total cases $\times 100 \%$ [19]. OS was the study endpoint and was defined as the period of time between treatment and either death or last follow-up.

\section{Statistical analyses}

SPSS 21.0 (SPSS Inc., Chicago, IL, USA) was utilize for all testing. Quantitative results are given as means \pm standard deviation, and t-tests was used to compare these values. Qualitative results are given as a number (\%), and Pearson Chi-Squared tests or Fisher's exact tests were employed for comparing these results as appropriate. Paired sample t-tests were used for comparison of ALBI scores and pre- and post-operative portal venous pressure in group A patients. Survival was analyzed via the Kaplan-Meier approach and through log-rank tests. $\mathrm{P}<0.05$ was the significance threshold. Cox univariate regression 
analyses were used to assess the relationship between specific factors and treatment outcomes. Multivariate logistic regression analyses were conducted based on the variables in the univariate analysis $(P<0.10)$.

\section{Results}

\section{Baseline information}

Baseline characteristics of the 53 patients in this study are shown in Table 1, with no significant clinicallyrelevant differences between groups. For group A patients, on average $18.2 \pm 1.7$ (range: 16-20) ${ }^{125}$ seeds were implanted, with mean radioactivity of $12.6 \mathrm{mCi} \pm 1.2$ (range: $11.2-14.0 \mathrm{mCi}$ ). Estimated radiation doses were roughly 40 - $50 \mathrm{~Gy}$, with a computerized treatment planning program (FTT Technology Ltd. Co, Beijing, China) being used to determine specific dose reference points.

\section{Complications and clinical outcomes}

There were no observed instances of abdominal hemorrhage, biloma, liver abscess, stent migration, or puncture site bleeding following PVS and endovascular ${ }^{125}$ I seed-strip implantation in any patients. In these patients, ALBI scores did alter following treatment, changing from $-2.57 \pm 0.42$ to $-1.92 \pm 0.53$ $(P<0.001)$. TACE-S-related AEs did not differ significantly between groups $(P>0.05)($ Table 2$)$.

Preoperative ALBI scores were comparable between groups $(P=0.724)$, nor did these scores differ 1month postoperatively $(P=0.666)$. However, at $3(P=0.007)$ and $6(P=0.044)$ months postoperatively, these scores were significantly different (Table 3 ). DCR was $71.4 \%$ in group $A$, while it was $44.0 \%$ in group $B$ $(P=0.043)$ after 6 months of treatment. Group A had a median survival of $342.0 \pm 20.5$ days [95\% $\mathrm{Cl}$ : 301.8 - 382.2 days], while in group $B$ it was $231.0 \pm 25.0$ days [95\%Cl: $182.0-280.0$ days] $(P=0.007)$. A stratified analysis demonstrated that median survival in those with type II PVTT was $313.0 \pm 60.1$ days [95\%Cl: 195.2 - 430.8 days] and $321.0 \pm 52.2$ days [95\% Cl: $218.7-423.3$ days] in groups A and $B$, respectively $(P=0.689)$, whereas in type III PVTT patients these survival times were $344.0 \pm 22.6$ days [95\% Cl: $300.0-388.4$ days] and $226.0 \pm 26.0$ days [95\% Cl: $175.0-277.0$ days], respectively $(\mathrm{P}=0.002)$ (Fig. 2).

Univariate Cox model analyses suggested that tumor size and number were both variables potentially associated with treatment outcomes, and as such, these were incorporated into a multivariate model. This analysis, in turn, determined that tumor size $>10 \mathrm{~cm}$ was an independent predictor of poor prognosis $(\mathrm{P}=0.002)$, whereas PVS-125 - -TACE-S was a predictor of a favorable patient prognosis $(P=0.040)$ (Table 4).

\section{Discussion}

HCC often readily undergoes portal vein invasion, leading to the development of PVTT, which can partially or fully obstruct the vein, resulting in increased venous pressure, decreased perfusion, and ultimately 
impaired liver function. The resultant elevation in pressure within the portal vein is also often associated with esophageal or stomach fundus bleeding. For these reasons, HCC patients with PVTT often have a poor prognosis [20]. While liver resection can cure some patients with type I-III HCC [21,22]. However, these patients typically exhibit poor long-term survival following surgery as HCC commonly recurs, particularly in the case of early recurrence within 1 year post-surgery [23].

One approach to improving HCC/PVTT patient OS is radiofrequency ablation, but because PVTT is near the bile duct, this approach has a significant potential to cause injury to either this structure or to the portal vein itself [24]. Also, transarterial radioembolization (TARE) has been used more frequently in patients with HCC and PVTT due to the decreased risk of hepatic ischemia and infarction, but it remains unavailable in commercial contexts in Japan and mainland China [25]. Benefits of stereotactic body radiotherapy (SBRT) include the fact that tumors can receive higher direct radiation doses while organs are protected from substantial radiation. However, lesion size is the main limiting factor for this approach, with local control rates ranging from $91 \%$ ( $<5 \mathrm{~cm}$ tumors) to $74 \%$ ( $\nabla 5 \mathrm{~cm}$ tumors) [26]. As such, many therapeutic strategies have been employed to treat patients suffering from HCC and PVTT, including TACE, sorafenib, and 3-dimensional conformal radiotherapy or combinations thereof. Even so, at present none of these approaches have been able to reliably and rapidly achieve desired treatment outcomes [27].

In recent years, brachytherapy has been widely utilized for tumor treatment. Due to the unique radiobiological characteristics of ${ }^{125}$ I seed, it effectively killed tumor cells without obvious damage to surrounding normal tissue [24]. In the present study, a significant survival benefit in patients in group $A$ relative to those in group B (342.0 vs 231.0 days). This may be because patients treated with the combination of PVS and endovascular ${ }^{125}$ I seed-strip implantation prior to TACE-S achieved better liver function, suggesting that restoring portal vein through and reduction of tumor thrombus burden is associated with improved OS $[12,13]$. With respect to safety, the most frequent AEs following the combination of PVS and ${ }^{125}$ I seed-strip implantation was a transient decrease in liver functionality, likely as a result of injury to the bile duct upon puncture of the portal vein [28]. TACE-S-associated adverse events for the two groups did not significantly differ. Grade 1/2 AEs were mild and alleviated through symptomatic treatment. Grade 3 and 4 AEs were managed in a conservative manner, temporarily reducing or ceasing sorafenib dosing as appropriate. Together these findings suggest that PVS combined with endovascular implantation ${ }^{125}$ I seed-strip followed by TACE-S is a well-tolerated intervention strategy.

Preoperative ALBI scores were comparable between groups $(P=0.724)$, nor did these scores differ 1month postoperatively $(P=0.666)$. However, at $3(P=0.007)$ and $6(P=0.044)$ months postoperatively, these scores were significantly different. PVS is well known to provide immediate restoration of blood flow to an obstructed portal vein, improving the hepatic blood supply. In addition, as ${ }^{125}$ I seed-strip were used for sustained intravascular brachytherapy, they may be a suitable means of counteracting neointimal hyperplasia and improving stent patency duration [28]. Together, these therapeutic interventions may thus be able to better ensure prolonged liver functionality following repeated TACE and long-term oral 
sorafenib intake. In contrast, group B patients only treated by TACE-S, where the embolization is linked to a higher risk of ischemic necrosis of healthy tissue, and chemotherapy can additionally aggravate remaining liver function [29]. And sorafenib itself also has the potential to reduce portal blood flow, elevating the risk of liver failure [30].

Also, a better disease control in group A than in group B (71.4\% vs $44.0 \%)$ after 6 months of treatment. TACE is reported to block flow through arteries that supply blood to the tumor, thus allowing for control of tumor growth and PVTT progression as the hepatic artery is the primary source of blood for tumor cells and thrombi [17]. As this region has blood supplied by both the artery and portal vein, however, and due to the potential for collateral arterial development, complete local necrosis is often difficult to achieve[31,32]. TACE is also linked to increased levels of vascular endothelial growth factor (VEGF), resulting in a higher risk of local recurrence [31]. While sorafenib combination treatment can reduce TACEassociated risks and improve outcomes, there are reports suggesting that sorafenib is less effective in those with Vp3/4 PVTT ( $<10 \%$ response rate) $[11,33]$. In contrast, ${ }^{125}$ I seed implantation in the portal vein can allow for effective PVTT control owing to sustained low-dose $x$ - and $y$-ray release throughout the tumor area, damaging tumor cell DNA and disrupting proliferation [24,34].

In a subgroup analysis, a significant difference in group A patient OS only for those with type III PVTT (344.0 vs 226.0 days). This may be due to the fact that near-total portal vein blockage in type III PVTT patients is linked to a rapid decrease in liver function [35]. And the liver function can be aggravated by repeated TACE and long-term sorafenib, as described above. This, in turn, requires modifications to or discontinuations of TACE-S treatment regimens, thus potentially constraining their therapeutic value $[36,37]$. Patients with PVTT also often died of acute variceal bleeding rather than due to direct damage from the liver tumor itself [32]. The PVS $/{ }^{125}$ I seed implantation strategy can improve portal vein obstruction, thereby reducing the risk of esophageal and gastric bleeding, thus reducing the risk of this indirect cause of death $[38,39]$. In addition, ${ }^{125}$ I seed implantation can improve local tumor control and delay PVTT progression, thus likely reducing the risk of local and distant metastasis [15].

No significant differences in OS for type II PVTT patients in these two treatment groups (313.0 vs 321.0 days), despite better liver function scores for group A patients. This may be because those in group A had a larger tumor burden, that tumor size $>10 \mathrm{~cm}$ was an independent predictor of poor prognosis $(P=0.002)$. This further suggests that tumor burden is a key prognostic indicator in HCC patients with PVTT and must be considered during treatment planning [40].

There are certain limitations to this study. For one, sample size was small, and while these results were statistically significant, a future larger longitudinal study would be of value for confirming these findings. This was also a retrospective analysis, and as such future randomized controlled trials will be essential in order to validate these findings. Lastly, a skin surface dosimeter was not employed after ${ }^{125}$ I seed-strip implantation, making it impossible to determine the exact radiation doses administered to patients in this study. 


\section{Conclusions}

The combination of PVS and endovascular ${ }^{125}$ I seed-strip implantation followed by TACE-S represents a safe and effective treatment for HCC patients suffering from type III PVTT, potentially being more effective than TACE-S alone.

\section{Abbreviations}

HCC: Hepatocellular carcinoma; PVTT: Portal vein tumor thrombus; BCLC: Barcelona Clinic Liver Cancer; OS: Overall survival; TACE: Transcatheter arterial chemoembolization; PVS: Portal vein stent; CT: Computed tomography; MRI: Magnetic resonance imaging; ECOG: Eastern Cooperative Oncology Group; PVA: Polyvinyl alcohol; AEs: Adverse events; CTCAE: Common Terminology Criteria for Adverse Events; ALBI: Albumin-Bilirubin; mRECIST: Modified Response Evaluation Criteria in Solid Tumors; CR: Complete response; PR: Partial response; SD: Stable disease; PD: Progressive disease; DCR: Disease control rate.

\section{Declarations}

\section{Ethics approval and consent to participate}

This study received approval from the ethics committee of our institution (LDYYLL2019-204), and all participants gave written informed consent to participate.

\section{Consent for publication}

Not applicable.

\section{Availability of data and materials}

Research data are stored in an institutional repository and will be shared upon request to the corresponding author.

\section{Competing interests}

The authors declare that they have no competing interests.

\section{Funding}

This work was supported in part by the Support Program for Longyuan Youth and Fundamental Research Funds for the Universities of Gansu Province (CN).

\section{Authors' contributions}

Conception and design of the study: Wenhui Wang, Shuangxi Li, Baohua Li. Lei Li kindly provided statistical advice for this manuscript. Generation, collection, assembly, analysis and/or interpretation of 
data: Fangyu $\mathrm{Xu}, \mathrm{Xujun}$ Yang. Drafting or revision of the manuscript and literature analysis/search: Fangyu Xu, Wenhui Wang. Approval of the final version of the manuscript: Wenhui Wang.

\section{Acknowledgements}

Not applicable.

\section{References}

1. Park JW, Kim YJ, Kim DY, et al. Sorafenib with or without concurrent transarterial chemoembolization in patients with advanced hepatocellular carcinoma: a phase III STAH trial. J Hepatol. 2019;70(4):684-691.

2. Sun J, Yang L, Shi J, et al. Postoperative Adjuvant IMRT for Patients with HCC and Portal Vein Tumor Thrombus: An Open-Label Randomized Controlled Trial. Radiother Oncol. 2019;140:20-25.

3. Li T, Yu Y, Liu J, et al. PIVKA-II level is correlated to development of portal vein tumor thrombus in patients with HBV-related hepatocellular carcinoma. Infect Agent Cancer. 2019;14:13.

4. Zhang XP, Gao YZ, Chen ZH, et al. An Eastern Hepatobiliary Surgery Hospital/Portal Vein Tumor Thrombus Scoring System as an Aid to Decision Making on Hepatectomy for Hepatocellular Carcinoma Patients with Portal Vein Tumor Thrombus: A Multicenter Study. Hepatology. 2019;69(5):2076-2090.

5. Sun JX, Shi J, Li N, et al. Portal vein tumor thrombus is a bottleneck in the treatment of hepatocellular carcinoma. Cancer Biol Med. 2016;13(4):452-458.

6. Zhu K, Chen J, Lai L, et al. Hepatocellular carcinoma with portal vein tumor thrombus: treatment with transarterial chemoembolization combined with sorafenib-a retrospective controlled study. Radiology. 2014;272(1):284-93.

7. Llovet JM, Ricci S, Mazzaferro V, et al. Sorafenib in advanced hepatocellular carcinoma. N Engl J Med. 2008;359(4):378-390.

8. Cheng AL, Kang YK, Chen Z, et al. Efficacy and safety of sorafenib in patients in the Asia-Pacific region with advanced hepatocellular carcinoma: a phase III randomised, double-blind, placebocontrolled trial. Lancet Oncol. 2009;10(1):25-34.

9. Zhang XP, Gao YZ, Chen ZH, et al. In-hospital Mortality after Surgical Resection in Hepatocellular Carcinoma Patients with Portal Vein Tumor Thrombus. 2019;10(1):72-80.

10. Wang K, Guo WX, Chen MS, et al. Multimodality Treatment for Hepatocellular Carcinoma with Portal Vein Tumor Thrombus: A Large-Scale, Multicenter, Propensity Mathching Score Analysis. Medicine (Baltimore). 2016;95(11):e3015.

11. Pan T, Li XS, Xie QK, et al. Safety and efficacy of transarterial chemoembolization plus sorafenib for hepatocellular carcinoma with portal venous tumour thrombus. Clin Radiol. 2014;69(12):e553-61.

12. Zhang ZH, Liu QX, Zhang W, et al. Combined endovascular brachytherapy, sorafenib, and transarterial chemobolization therapy for hepatocellular carcinoma patients with portal vein tumor 
thrombus. World J Gastroenterol. 2017;23(43):7735-7745.

13. Sun JH, Zhou T, Zhu T, et al. Portal Vein Stenting Combined with lodine-125 Seeds Endovascular Implantation Followed by Transcatheter Arterial Chemoembolization for Treatment of Hepatocellular Carcinoma Patients with Portal Vein Tumor Thrombus. Biomed Res Int. 2016;2016:3048261.

14. Lu J, Guo JH, Zhu HD, Zhu GY, Chen L, Teng GJ. Safety and Efficacy of Irradiation Stent Placement for Malignant Portal Vein Thrombus Combined with Transarterial Chemoembolization for Hepatocellular Carcinoma: A Single-Center Experience. J Vasc Interv Radiol. 2017;28(6):786-794.

15. Sun H, Zhang M, Liu R, Liu Y, Hou Y, Wu C. Endovascular implantation of 125 l seed combined with transcatheter arterial chemoembolization for unresectable hepatocellular carcinoma. Future Oncol. 2018;14(12):1165-1176.

16. Li S, Li L, Li B, Wang W. Safety and efficacy of endovascular implantation of a portal vein stent combined with iodine-125 seed-strips followed by transcatheter arterial chemoembolization with sorafenib for the treatment of hepatocellular carcinoma with portal vein tumor thrombosis. $\mathrm{Br} \mathrm{J}$ Radiol. 2020;93(1112):20190279.

17. Zhang W, Liu C, Tan Y, et al. Albumin-Bilirubin Score for Predicting Post-Transplant Complications Following Adult-to-Adult Living Donor Liver Transplantation. Ann Transplant. 2018;23:639-646.

18. Lu J, Guo JH, Zhu HD, Zhu GY, Chen L, Teng GJ. Safety and Efficacy of Irradiation Stent Placement for Malignant Portal Vein Thrombus Combined with Hepatocellular Carcinoma: A Single-Center Experience. J Vasc Interv Radiol. 2017;28(6):786-794.

19. Lv WF, Liu KC, Lu D, et al. Transarterial chemoembolization for hepatocellular carcinoma combined with portal vein tumor thrombosis. Cancer Manag Res. 2018;10:4719-4726.

20. Liu PH, Huo TI, Miksad RA. Hepatocellular Carcinoma with Portal Vein Tumor Involvement: Best Management Strategies. Semin Liver Dis. 2018;38(3):242-251.

21. Kokudo T, Hasegawa K, Matsuyama Y, et al. Survival benefit of liver resection for hepatocellular carcinoma associated with portal vein invasion. J Hepatol. 2016;65(5):938-943.

22. Wang JC, Xia AL, Xu Y, Lu XJ. Comprehensive treatments for hepatocellular carcinoma with portal vein tumor thrombosis. J Cell Physiol. 2019;234(2):1062-1070.

23. Chen ZH, Zhang XP, Zhou TF, et al. Adjuvant transarterial chemoembolization improves survival outcomes in hepatocellular carcinoma with microvascular invasion: A systematic review and metaanalysis. Eur J Surg Oncol. 2019;45(11):2188-2196.

24. Yuan D, Gao Z, Zhao J, Zhang H, Wang J. 125I seed implantation for hepatocellular carcinoma with portal vein tumor thrombus: A systematic review and meta-analysis. Brachytherapy. 2019;184(4):521-529.

25. Lu J, Zhang XP, Zhong BY, et al. Management of patients with hepatocellular carcinoma and portal vein tumour thrombosis: comparing east and west. Lancet Gastroenterol Hepatol. 2019;4(9):721-730.

26. Lee J, Shin IS, Yoon WS, Koom WS, Rim CH. Comparisons between radiofrequency ablation and stereotactic body radiotherapy for liver malignancies: Meta-analyses and a systematic review. Radiother Oncol. 2020;145:63-70. 
27. Tan T, Xiao Y, Zhou S, Ma C, Zhang Z. Y-configuration stent combined with iodine- 125 seeds strand for the treatment of hepatocellular carcinoma with tumor thrombosis in portal vein branches: A case report. Medicine (Baltimore). 2017;96(46):e8660.

28. JianJun Luo, ZhiPing Yan, QinXin Liu, XuDong Qu, and JianHua Wang. Endovascular Placement of lodine-125 Seed Strand and Stent Combined with Chemoembolization for Treatment of Hepatocellular Carcinoma with TumorThrombus in Main Portal Vein. J Vasc Interv Radiol. 2011;22(4):479-89.

29. Zhang YF, Shang H, Zeng XL, Ji H, Li YM, Lu HW. Postoperative adjuvant chemo (embolization) therapy for hepatocellular carcinoma with portal vein tumor thrombosis. Onco Targets Ther. 2018;11:5407-5417.

30. Yamasaki A, Umeno N, Harada S, Tanaka K, Kato M, Kotoh K. Deteriorated portal flow may cause liver failure in patients with hepatocellular carcinoma being treated with sorafenib. J Gastrointest Oncol. 2016;7(3):E36-40.

31. Miki I, Murata S, Uchiyama F, et al. Evaluation of the relationship between hepatocellular carcinoma location and transarterial chemoembolization efficacy. World J Gastroenterol. 2017;23(35):64376447.

32. Sun J, Shi J, Huang B, et al. The degree of hepatic arterial blood supply of portal vein tumor thrombus in patients with hepatocellular carcinoma and its impact on overall survival after transarterial chemoembolization. Oncotarget. 2017;8(45):79816-79824.

33. Chan SL, Chong CC, Chan AW, Poon DM, Chok KS. Management of hepatocellular carcinoma with portal vein tumor thrombosis: Review and update at 2016. World J Gastroenterol. 2016;22(32):7289300 .

34. Lin J, Jiang H, Yang W, et al. Predictive factors of benefit from iodine-125 brachytherapy for hepatocellular carcinoma with portal vein tumor thrombosis. Brachytherapy. 2019;182(2):233-239.

35. Su F, Chen KH, Liang ZG, et al. Comparison of three-dimensional conformal radiotherapy and hepatic resection in hepatocellular carcinoma with portal vein tumor thrombus. Cancer Med. 2018;7(9):43874395.

36. Sun Hong Yoo, Jeong Won Jang, Jung Hyun Kwon, Seung Min Jung, Bohyun Jang, and Jong Young Choi. Preemptive antiviral therapy with entecavir can reduce acute deterioration of hepatic function following transarterial chemoembolization. Clin Mol Hepatol. 2016;22(4):458-465.

37. Chang WT, Lu SN, Rau KM, Huang CS, Lee KT. Increased cumulative doses and appearance of handfoot skin reaction prolonged progression free survival in sorafenib-treated advanced hepatocellular carcinoma patients. Kaohsiung J Med Sci. 2018;34(7):391-399.

38. Chuan-Xing L, Xu H, Bao-Shan H, et al. Efficacy of therapy for hepatocellular carcinoma with portal vein tumor thrombus: Chemoembolization and stent combined with iodine-125 seed. Cancer Biol Ther. 2011;12(10):865-71.

39. Wu YF, Wang T, Yue ZD, et al. Stents combined with iodine-125 implantation to treat main portal vein tumor thrombus. World J Gastrointest Oncol. 2018;10(12):496-504. 
40. Wang Q, Xia D, Bai W, et al. Development of a prognostic score for recommended TACE candidates with hepatocellular carcinoma: A multicentre observational study. J Hepatol. 2019;70(5):893-903.

\section{Tables}

Table 1. Patient demographic and clinical characteristics 


\begin{tabular}{|c|c|c|c|}
\hline Variable & Group A $(n=28)$ & Group B $(n=25)$ & P-value \\
\hline Sex, n (\%) & & & $0.857^{*}$ \\
\hline Male & $23(82.1)$ & $21(84.0)$ & \\
\hline Female & $5(17.9)$ & $4(16.0)$ & \\
\hline Age (year), mean \pm SD & $57.3 \pm 12.2$ & $57.5 \pm 8.0$ & $0.956 \#$ \\
\hline Child-Pugh score, n (\%) & & & $0.925^{\star}$ \\
\hline 5 & $17(60.7)$ & $15(60.0)$ & \\
\hline 6 & $8(28.6)$ & $8(32.0)$ & \\
\hline 7 & $3(10.7)$ & $2(8.0)$ & \\
\hline ECOG performance, $\mathrm{n}(\%)$ & & & $0.991 *$ \\
\hline 1 & $19(67.9)$ & $17(68.0)$ & \\
\hline 2 & $9(32.1)$ & $8(32.0)$ & \\
\hline Etiology, n (\%) & & & $0.902^{*}$ \\
\hline HBV & $23(82.1)$ & $20(80.0)$ & \\
\hline $\mathrm{HCV}$ & $3(10.7)$ & $2(8.0)$ & \\
\hline Alcohol & $1(3.6)$ & $2(8.0)$ & \\
\hline Other & $1(3.6)$ & $1(4.0)$ & \\
\hline Classifications of PVTT, n (\%) & & & $0.983^{*}$ \\
\hline 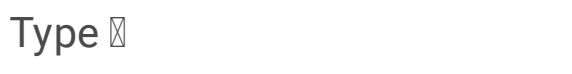 & $10(35.7)$ & $9(36.0)$ & \\
\hline 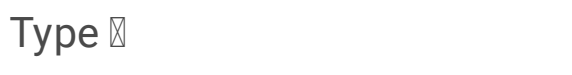 & $18(64.3)$ & $16(64.0)$ & \\
\hline AFP (ng/mL), n (\%) & & & $0.958^{*}$ \\
\hline$>400$ & $11(39.3)$ & $10(40.0)$ & \\
\hline$\leq 400$ & $17(60.7)$ & $15(60.0)$ & \\
\hline Ascites, n (\%) & & & $0.786^{*}$ \\
\hline Present & $8(28.6)$ & $8(32.0)$ & \\
\hline Absent & $20(71.4)$ & $17(68.0)$ & \\
\hline TACE times, mean \pm SD & $2.8 \pm 0.9$ & $1.8 \pm 0.8$ & $\otimes 0.001 \#$ \\
\hline Tumor size $(\mathrm{cm})$, mean \pm SD & $9.0 \pm 3.5$ & $9.2 \pm 2.6$ & $0.837 \#$ \\
\hline Tumor size, n (\%) & & & $0.834^{\star}$ \\
\hline
\end{tabular}




\begin{tabular}{|llll|}
$\geq 10 \mathrm{~cm}$ & $10(35.7)$ & $9(36.0)$ & \\
\hline$<10 \mathrm{~cm}$ & $18(64.3)$ & $16(64.0)$ & \\
\hline Multiple tumors, $\mathrm{n}(\%)$ & & & \\
\hline$\geq 3$ & $9(32.1)$ & $8(32.0)$ & \\
\hline$<3$ & $19(67.9)$ & $17(68.0)$ & \\
\hline Fistula, $\mathrm{n}(\%)$ & & & $0.806^{*}$ \\
\hline Present & $4(14.3)$ & $3(12.0)$ & \\
\hline Absent & $24(85.7)$ & $22(88.0)$ & \\
\hline Total bilirubin $(\mu \mathrm{mol} / \mathrm{L})$, mean $\pm \mathrm{SD}$ & $24.7 \pm 10.4$ & $25.3 \pm 11.1$ & $0.833 \#$ \\
\hline
\end{tabular}

*Indicates data obtained with Pearson Chi-Square test; \# indicates data obtained with independent sample t-test. ECOG, Eastern Cooperative Oncology Group; HBV, hepatitis B virus; HCV, hepatitis C virus; PVTT, portal vein tumor thrombus; AFP, a-Fetoprotein; TACE, transcatheter arterial chemoembolization. $P<0.05$ was the significance threshold.

Table 2. TACE-S-associated adverse events for the two groups (\%)

\begin{tabular}{|llllll|}
\hline \multirow{2}{*}{ Complications } & \multicolumn{2}{l}{ Group A (n=28) } & \multicolumn{2}{l}{ Group B (n=25) } & \multirow{2}{*}{ P-value } \\
\cline { 2 - 5 } & Grade 1-2 & Grade 3-5 & Grade 1-2 & Grade 3-5 & \\
\hline Nausea or vomiting & $49.2 \%$ & 0 & $48.0 \%$ & 0 & 0.707 \\
\hline Fever & $35.7 \%$ & 0 & $32.0 \%$ & 0 & 0.776 \\
\hline Abdominal pain & $39.3 \%$ & 0 & $44.0 \%$ & 0 & 0.728 \\
\hline Abscess & 0 & $3.6 \%$ & 0 & 0 & 0.340 \\
\hline Fatigue & $25.0 \%$ & $7.1 \%$ & $28.0 \%$ & $4.0 \%$ & 0.871 \\
\hline Diarrhea & $35.7 \%$ & $7.1 \%$ & $32.0 \%$ & $88.0 \%$ & 0.958 \\
\hline Hypertension & $32.1 \%$ & $3.6 \%$ & $32.0 \%$ & $4.0 \%$ & 0.997 \\
\hline Hand-foot syndrome & $42.9 \%$ & $14.3 \%$ & $36.0 \%$ & $16.0 \%$ & 0.878 \\
\hline Alopecia & $7.1 \%$ & 0 & $8.0 \%$ & 0 & 0.906 \\
\hline Pruritus & $14.3 \%$ & 0 & $20.0 \%$ & 0 & 0.580 \\
\hline Rash or desquamation & $7.1 \%$ & $3.6 \%$ & $8.0 \%$ & $4.0 \%$ & 0.989 \\
\hline Voice change & $3.6 \%$ & 0 & 0 & 0 & 0.340 \\
\hline Anorexia & $14.3 \%$ & 0 & $12.0 \%$ & 0 & 0.806 \\
\hline
\end{tabular}


TACE-S, transcatheter arterial chemoembolization-sorafenib. $\mathrm{P}<0.05$ was the significance threshold.

Table 3. Pre- and post-operative ALBI scores in the two treatment groups

\begin{tabular}{|llll|}
\hline ALBI score & Group A $(n=28)$ & Group B $(n=25)$ & P-value \\
\hline Number of assessed patients & 28 & 25 & \\
\hline Preoperative & $-2.57 \pm 0.42(n=28)$ & $-2.61 \pm 0.38$ & 0.724 \\
\hline Number of assessed patients & 28 & 25 & \\
\hline 1 months postoperative & $-2.62 \pm 0.46(n=28)$ & $-2.20 \pm 0.59$ & 0.666 \\
\hline Number of assessed patients & 28 & 25 & \\
\hline 3 months postoperative & $-2.17 \pm 0.59(n=28)$ & $-1.69 \pm 0.48$ & $* 0.007$ \\
\hline Number of assessed patients & 24 & 21 & \\
\hline 6 months postoperative & $-2.28 \pm 1.23(n=24)$ & $-1.47 \pm 0.31$ & $* 0.044$ \\
\hline
\end{tabular}

* indicates significance. ALBI, Albumin-Bilirubin.

Table 4. Univariate and multivariate analyses of variables associated with OS in HCC patients suffering from PVTT 


\begin{tabular}{|c|c|c|c|c|}
\hline \multirow[t]{2}{*}{ Variables } & $\begin{array}{l}\text { Univariate } \\
\text { analysis }\end{array}$ & \multirow[t]{2}{*}{ P-value } & $\begin{array}{l}\text { Multivariate } \\
\text { analysis }\end{array}$ & \multirow[t]{2}{*}{$\begin{array}{l}\mathrm{P}- \\
\text { value }\end{array}$} \\
\hline & $\mathrm{HR}(95 \% \mathrm{Cl})$ & & $\mathrm{HR}(95 \% \mathrm{Cl})$ & \\
\hline Gender (male vs female) & $\begin{array}{l}1.476(0.710- \\
3.068)\end{array}$ & 0.298 & - & - \\
\hline ECOG scores (1 vs 2) & $\begin{array}{l}1.170(0.646- \\
2.119)\end{array}$ & 0.604 & - & - \\
\hline $\begin{array}{l}\text { Aetiology } \\
\text { (HBV/HCV/Alcohol/Other) }\end{array}$ & $\begin{array}{l}0.846(0.583- \\
1.228)\end{array}$ & 0.379 & - & - \\
\hline Child-Pugh score (5/6/7) & $\begin{array}{l}1.205(0.812- \\
1.787)\end{array}$ & 0.355 & - & - \\
\hline $\operatorname{AFP}(\leq 400$ vs $>400 \mathrm{ng} / \mathrm{mL})$ & $\begin{array}{l}0.672(0.378- \\
1.193)\end{array}$ & 0.175 & - & - \\
\hline Ascites (absent vs present) & $\begin{array}{l}1.014(0.562- \\
1.832)\end{array}$ & 0.962 & - & - \\
\hline Tumorsize $(\leq 10 \mathrm{~cm}$ vs > $10 \mathrm{~cm})$ & $\begin{array}{l}3.575(1.836- \\
6.962)\end{array}$ & $\begin{array}{l}*< \\
0.001\end{array}$ & $2.920(1.478-5.768)$ & * 0.002 \\
\hline Multiple tumors ( $<3$ vs $\geq 3$ ) & $\begin{array}{l}1.897(1.028- \\
3.500)\end{array}$ & * 0.041 & - & - \\
\hline 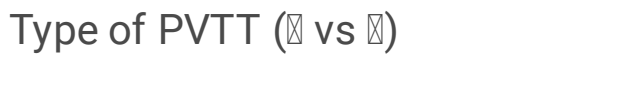 & $\begin{array}{l}1.422(0.783- \\
2.583)\end{array}$ & 0.247 & - & - \\
\hline Fistula (absent vs present) & $\begin{array}{l}1.006(0.449- \\
2.252)\end{array}$ & 0.988 & - & - \\
\hline Treatment strategy & $\begin{array}{l}0.576(0.332- \\
1.002)\end{array}$ & 0.510 & $0.547(0.308-0.972)$ & ${ }^{*} 0.040$ \\
\hline
\end{tabular}

* indicates significance. HCC, hepatocellular carcinoma; PVTT, portal vein tumor thrombus; ECOG, Eastern Cooperative Oncology Group; HBV, hepatitis B virus; HCV, hepatitis C virus; AFP, a-Fetoprotein.

\section{Figures}




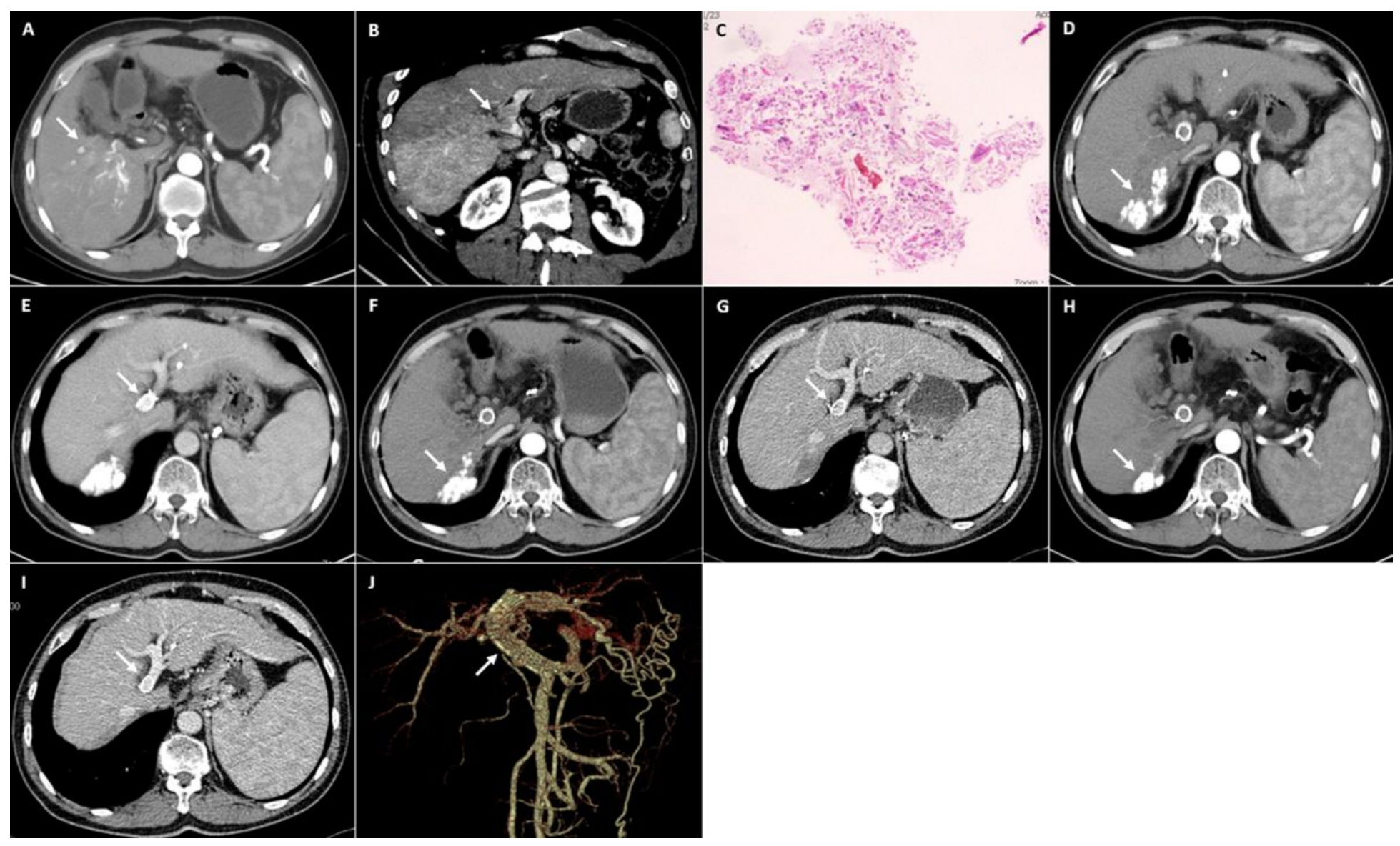

Figure 1

Results from a 63-year-old male who had HCC with type III PVTT. (A-B) Contrast enhanced CT scan exhibiting a hepatic arterial phase hyperattenuation lesion at segment 6 , segment 7 , and PVTT extending to the main portal vein. (C) Diaminobenzidine (DAB) staining used to establish a definitive PVTT pathological diagnosis. (D-E) Results from a 3-month follow-up following the combination of PVS-125ITACE-S; lipiodol accumulation in the tumor and satisfactory patency of the stent was observed. (F-G) Results from 6-month follow-up; the treated lesion was decreased in size and satisfactory patency of the stent was observed. $(\mathrm{H}-\mathrm{I})$ Results from 12-month follow-up; the treated lesion was continuing to decrease in size, and satisfactory patency of the stent was observed. $(\mathrm{J})$ Meanwhile, vascular reconstruction showed stent and 125I seed-strip were implanted correctly in the portal vein without displacement.
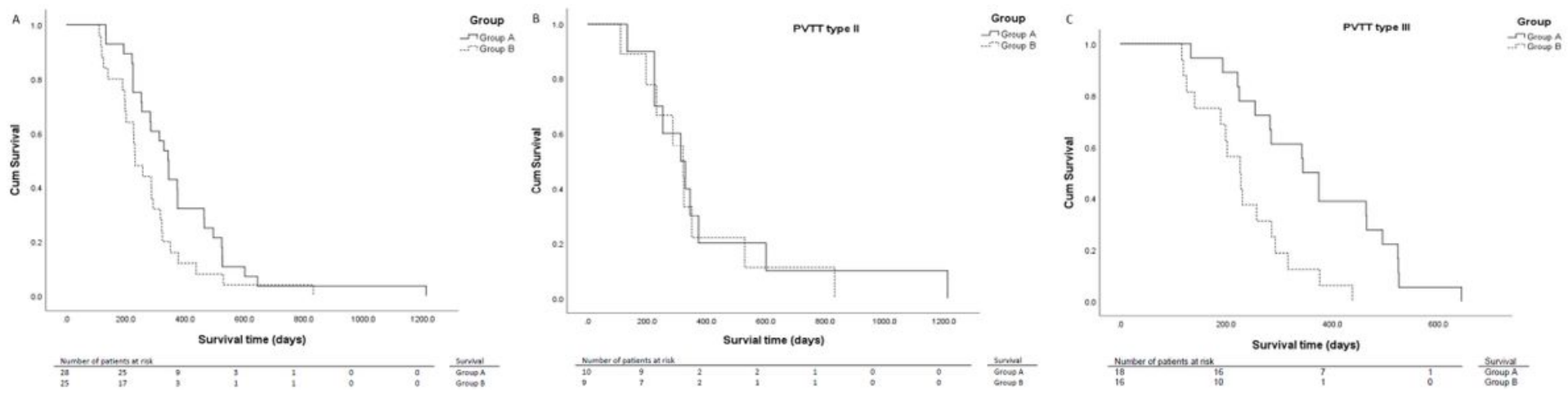

Figure 2 
Kaplan-Meier OS curves from HCC patients suffering from PVTT treated via a combination of PVS-125ITACE-S (Group A) or via TACE-S alone (Group B). (A) The entire study population ( $P=0.007)$. (B) Type II PVTT patients $(P=0.689)$. (C) Type III PVTT patients $(P=0.002)$. 